\title{
Indications and Limitations of Creatinine in the Diet of Athletes
}

Edison Ikonomi* and Anesti Qeleshi

Sport University Tirana, Albania

ISSN: 2577-1914

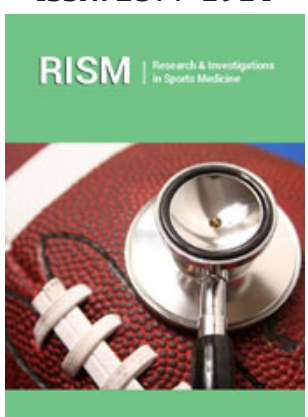

*Corresponding author: Edison Ikonomi, Sport University Tirana, Albania

Submission: 眥 May 05, 2020

Published: 鲇July 24, 2020

Volume 6 - Issue 5

How to cite this article: Edison Ikonomi, Indications and Limitations of Creatinine in the Diet of Athletes. Res Inves Sports Med, 6(5): RISM.000646. 2020. DOI: $10.31031 /$ RISM.2020.06.000646

Copyright@ Edison Ikonomi, This article is distributed under the terms of the Creative Commons Attribution 4.0 International License, which permits unrestricted use and redistribution provided that the original author and source are credited.

\section{Short Communication}

Creatinine (C) is present almost entirely in muscle (95\%), in free form (30-40\%) and phosphorylated (CP). Other organs where creatinine is found are heart, brain, testicles, retina of eyes. Increased P Cr concentration is expressed with greater ATP production during maximum physical activity.

\section{Biochemical Aspects}

Creatinine is a natural substance present in significant amounts in foods and is incorrectly classified as a medicament. The creatinine load phase brings about an increase in body weight by $0.5-1.5 \mathrm{~kg}$, which is mainly related to water retention as intra and extracellular. In a male weighing $70 \mathrm{~kg}$, the total creatinine is estimated to be $120 \mathrm{~g}$, oscillating from 90 $160 \mathrm{mg}$. Turnover (daily loss) is about $1.5 \%$, which corresponds to about $2 \mathrm{~g}$ per day. This loss is replaced by both endogenous synthesis (in the liver, pancreas, kidney) starting from glycine and arginine (and methionine) as well as from the diet, mainly meat and fish. In vegetarians, the daily loss of creatinine is replaced by endogenous site-dependent regulated food.

\section{Different Mechanisms in Improving Muscular Performance}

The increased concentration of $\mathrm{P} \mathrm{Cr}$ is expressed with greater production of ATP during maximal physical effusions. During the break between short and intense exercises, P Cr resynthesis of activated glycogen action and other catabolic pathways appears to increase. Since the hydrolysis of $\mathrm{P} \mathrm{Cr}$ consumes $\mathrm{H}$ ions, it affects the intracellular acidosis buffering during physical exercise. It can happen even hypertrophy and hyper-hydration of muscle cells.

\section{The Effects of Dietary Creatinine Supplementation on Muscle}

The muscle has a maximum creatinine capacity of $150-160 \mathrm{mmol} / \mathrm{kg}$. The lower the initial concentration, the greater the potential benefit of dietary supplementation. The average increase recorded by different "load" methods varies from 6 to $20 \%$. There is a significant proportion (20-30\%) of individuals who are unable to increase muscle creatinine concentration. Muscle concentration returns to basal values 4 weeks after discontinuation of nutritional supplementation $[1,2]$.

\section{Food Accompaniment}

It has been demonstrated that association with carbohydrates $(100 \mathrm{~g}$ for every $5 \mathrm{~g}$ of creatinine) improves absorption, possibly by being driven by insulin for its transport to the muscle. But recent studies have shown that muscle creatinine uptake (in vitro into isolated mouse muscle) is largely $\mathrm{Na}$ dependent, whereas insulin has no effect on creatinine uptake in muscle. One study has shown that caffeine can have an inhibitory effect on potential work performance improvement. In the three groups studied: placebo (P), creatinine (C) and creatinine plus caffeine $(\mathrm{C}+)$, groups $(\mathrm{C})$ and $(\mathrm{C}+)$ had a similar $(4-6 \%)$ increase in muscular phosphocreatine where only the group (C) increased by 10-20\% more knee extension force compared to the $(\mathrm{C}+)$ and $(\mathrm{P})$ groups. The mechanism of this inhibitory effect is not fully known. 


\section{Data on Muscular Physiology}

Creatinine supplementation does not stimulate any exerciserelated muscular adaptation. In fact, strength activity and aerobic or anaerobic capacity do not appear to significantly increase the levels of phosphocreatine, creatinine, and phosphocreatine kinase activity. Although muscular performance is significantly enhanced by the presence of the PC / C system, on the other hand this is not necessary as is ATP for muscle contraction. There is no evidence that creatinine directly stimulates muscle protein synthesis, so there seems to be no anabolic effect.

\section{Effects on Sport Performance}

The most pronounced improvements were mainly recorded in the last exercises ( 5 th to 6 th) of the series, when theywere performed at intervals of 20"- 60 " between exercises. The exercises performed were maximal, with such force that they could be performed in just a few seconds. Efficiency is related to the ratio between the amount of work performed and the time off: rest intervals, whether too long or too short, eliminate any kind of improvement in performance. If the recovery time is prolonged, the improvement "dilutes", in the sense that we are no longer dealing with a series of repetitions, but with special events, though very close to each other, in which the difference between the athletes "treated" and "untreated" is minimal. If the interval is too short, maximum creatinine concentration is not able to exert its effect on phosphocreatine re-synthesis. Almost all studies concur in the fact that there is no improvement in aerobic performance. Even weight gain, which usually occurs when taking creatinine as a dietary supplement, can impair aerobic performance in long-lasting exercises. Some studies, which still need deepening and confirmation, have shown that we can have a positive effect on short sprints performed during or after a prolonged physical exercise. Some authors oppose the use in competitions and exercises of laboratory studies, claiming that creatinine has shown a reasonable "ergogenic" benefit only in repeated laboratory cyclical efficiencies. In fact there is a lot of difference between the results and rarely have they been verified outside the laboratories. All this is likely related to the differences between laboratory conditions and real race conditions (only some sports include short exercises, repeated at intervals and requiring maximum strain), the existence of individuals who do not respond to treatment and although the literature is very wide, there are few serious studies, independent and reliable [3-5].

\section{Conclusion}

We can say that there is a real ergogenic effect from creatinine supplementation for short, intense and repetitive exercises. This improvement in performance seems to be related to the dose, the period of intake, the type of physical exercise, and the relationship between the work performed and the rest (recovery). For extended sports no effect has been demonstrated. It is still difficult to apply in sports practice the results achieved in the laboratory, also due to the fact that there is a variety of individual reactions to the treatment.

\section{References}

1. Armsey J, Thomas D, Gary A (1997) Nutrition supplements: science Vs hype. The Physician and Sports Medicine 25(6).

2. Brink WD (1996) what's in your Creatine?

3. Brzycki M (2000) Creatine supplementation: effective and safe? Master Trainer 10: 11-18.

4. Lulinski (1999) Creatine supplementation.

5. McDonald T, Drescher KM, Weber A, Tracy S (2012) Creatinine inhibits bacterial replication. J Antibiot (Tokyo) 65(3): 153-156.

For possible submissions Click below:

Submit Article 